\title{
Diacronie
}

Studi di Storia Contemporanea

$\mathrm{N}^{\circ} 36,4 \mid 2018$

Viaggi e turismo nell'Europa del Novecento

\section{Concilier dictature totalitaire et tourisme. Le régime fasciste italien face à ses ambitions touristiques}

\section{Christophe Poupault}

\author{
(2) OpenEdition \\ Journals \\ Édition électronique \\ URL : https://journals.openedition.org/diacronie/10134 \\ DOI : 10.4000/diacronie. 10134 \\ ISSN : 2038-0925 \\ Éditeur \\ Association culturelle Diacronie \\ Référence électronique \\ Christophe Poupault, «Concilier dictature totalitaire et tourisme. Le régime fasciste italien face à ses \\ ambitions touristiques ", Diacronie [En ligne], $N^{\circ} 36,4 \mid 2018$, document 6 , mis en ligne le 29 décembre \\ 2018, consulté le 17 février 2022. URL : http://journals.openedition.org/diacronie/10134 ; DOI : https:// \\ doi.org/10.4000/diacronie.10134
}




\section{Diacronie}

Studi di Storia Contemporanea

$36,4 / 2018$

Viaggi e turismo nell'Europa del Novecento

\section{Concilier dictature totalitaire et tourisme. Le régime fasciste italien face à ses ambitions touristiques}

Christophe POUPAULT

Per citare questo articolo:

POUPAULT, Christophe, «Concilier dictature totalitaire et tourisme. Le régime fasciste italien face à ses ambitions touristiques», Diacronie. Studi di Storia Contemporanea : Viaggi e turismo nell'Europa del Novecento, 36, 4/2018, $29 / 12 / 2018$

URL: < http://www.studistorici.com/2018/12/29/poupault_numero_36/ >

Diacronie Studi di Storia Contemporanea $\rightarrow$ http://www.diacronie.it Rivista storica online. Uscita trimestrale.

redazione.diacronie@hotmail.it

Comitato di direzione: Naor Ben-Yehoyada - João Fábio Bertonha - Christopher Denis-Delacour - Maximiliano Fuentes Codera Anders Granås Kjøstvedt - John Paul Newman - Deborah Paci - Niccolò Pianciola - Spyridon Ploumidis - Wilko Graf Von Hardenberg

Comitato di redazione: Jacopo Bassi - Luca Bufarale - Gianluca Canè - Luca G. Manenti - Fausto Pietrancosta - Alessandro Salvador - Matteo Tomasoni - Luca Zuccolo Creative Commons 3.0. Possono essere riprodotti e modificati a patto di indicare eventuali modifiche dei contenuti, di riconoscere la paternità dell'opera e di condividerla allo stesso modo. La citazione di estratti è comunque sempre autorizzata, nei limiti previsti dalla legge. 


\title{
6/ Concilier dictature totalitaire et tourisme. Le régime fasciste italien face à ses ambitions touristiques
}

\author{
Christophe POUPAULT
}

\begin{abstract}
L'Italie fasciste a toujours encouragé le tourisme pour des raisons économiques et politiques. Entre 1929 et 1933, plusieurs rapports révèlent néanmoins ses fragilités touristiques et proposent des solutions pour y remédier en améliorant ses équipements et l'accueil des étrangers, tout en conciliant l'ensemble avec les contraintes de la dictature. Les contrôles aux frontières, la surveillance, la qualité de l'hôtellerie, des transports, ou la tenue des bureaux touristiques à l'étranger retiennent particulièrement l'attention. Confrontés aux remarques des voyageurs, ces rapports permettent de saisir les insuffisances touristiques du régime fasciste, les réponses apportées pour les corriger, et leurs limites.
\end{abstract}

Les recherches sur l'histoire du tourisme en Italie ont bien mis en évidence le passage progressif du voyage érudit ou romantique, issu du Grand Tour, au tourisme de masse qui a bouleversé la société et obligé les pouvoirs publics à adapter leurs politiques ${ }^{1}$. Dans cette évolution, l'entre-deux-guerres correspond à une phase de transition où les déplacements ne sont plus uniquement l'apanage des élites fortunées ou de quelques privilégiés, sans être pour autant comparables aux flux qui caractérisent l'après Seconde Guerre mondiale. Si le régime fasciste, dans le cadre de la construction totalitaire ${ }^{2}$, a rapidement cherché à créer une culture de masse en encadrant les loisirs et le temps libre des Italiens $^{3}$, il s'est aussi consacré à la promotion du

\footnotetext{
${ }^{1}$ BERRINO, Annunziata, Storia del turismo in Italia, Bologna, Il Mulino, 2011 ; JELARDI, Andrea, Storia del viaggio e del turismo in Italia, Milano, Mursia, 2012; AVALLONE, Paola, STRANGIO, Donatella (a cura di), Turismi e turisti. Politica, innovazione, economia in Italia in età contemporanea, Milano, Franco Angeli, 2016.

${ }^{2}$ Sur le sujet, outre les travaux pionniers de Renzo de Felice, voir : LUPO, Salvatore, Le fascisme italien. La politique dans un régime totalitaire, Paris, Flammarion, 2003 ; GENTILE, Emilio, Qu'est-ce que le fascisme. Histoire et interprétation, Paris, Gallimard, 2004 ; ID., La voie italienne au totalitarisme. Le parti et l'État sous le régime fasciste, Monaco, Éditions du Rocher, 2004 ; DORMAGEN, Jean-Yves, Logiques du fascisme. L'État totalitaire en Italie, Paris, Fayard, 2008 ; GENTILE, Emilio (a cura di), Modernità totalitaria. Il fascismo italiano, Roma-Bari, Laterza, 2008.

${ }^{3}$ DE GRAZIA, Victoria, Consenso et cultura di massa nell'Italia fascista. L'organizzazione del dopolavoro, Roma-Bari, Laterza, 1981.
} 
tourisme, conscient que cette industrie nouvelle offrait des avantages économiques et politiques ${ }^{4}$. Ainsi, en 1934, sur 31 pays étudiés, la Société des Nations place l'Italie au troisième rang pour l'importance de ses entrées touristiques ${ }^{5}$, preuve que le pays, en dépit de la dictature, est attractif pour les visiteurs étrangers ${ }^{6}$.

Afin de contrôler et d'encadrer toutes les activités touristiques, l'État fasciste a progressivement cherché à fasciser les organisations déjà existantes, à l'instar du Touring Club italien $^{7}$ ou de l'ENIT (Ente nazionale per le industrie turistiche), tout en créant de nouveaux organismes qui sont en pleine expansion jusqu'à la guerre : Compagnie italienne de tourisme (CIT) en 1927, Commissariat pour le tourisme en avril 1931, remplacé en novembre 1934 par la direction générale du tourisme ${ }^{8}$, placée sous la tutelle du Secrétariat d'État à la Presse et à la Propagande, devenu ministère en 1935, avant son intégration en 1937 au ministère de la Culture populaire9 Toutefois, au-delà de cette centralisation, le régime est conscient des limites inhérentes à l'offre touristique de la péninsule, l'obligeant à adapter ses équipements et ses infrastructures à la concurrence étrangère, tout en conciliant les contraintes de la dictature avec les voyages étrangers.

Dès lors, au tournant des années trente, afin de continuer à attirer de nombreux visiteurs, d'améliorer leur bien-être et de diffuser auprès d'eux une image positive de l'Italie "nouvelle », plusieurs rapports officiels ou officieux, tous destinés à Mussolini, permettent de comprendre la façon dont les autorités fascistes ont souhaité moderniser les équipements italiens et accommoder les rigueurs de la dictature à leurs ambitions touristiques.

\footnotetext{
${ }^{4}$ CHAIX, Edmond, « Une industrie nouvelle. Le tourisme », in Revue des Deux Mondes, $1^{\mathrm{er}}$ janvier 1932, pp. 6393.

${ }^{5}$ PALOSCIA, Franco, Storia del turismo nell'economia italiana, Città di Castello, Petruzzi Editore, 1994, p. 19.

${ }^{6}$ En 1924, sur presque 1,3 million de touristes étrangers en Italie, 285406 sont des Allemands, 232623 sont originaires de l'ancienne Autriche-Hongrie (toujours prise en compte dans le recensement), 187057 sont des Anglais, 171609 des Américains et 115719 des Français (ENIT, Statistica del movimento turistico in Italia. Anno 1924, Roma, Tipografia del Senato, 1925, p. 60). En 1929, l'Italie reçoit 1,25 million de touristes, dont 334077 Allemands, 227570 Américains, 149523 Anglais et 103793 Français (ENIT, Relazione sull'attività svolta nell'anno 1929-VII, Roma, Castaldi, 1930, p. 81). En 1934, 3,14 millions de touristes voyagent dans la péninsule, le record étant atteint en 1937 avec 5,02 millions, avant une baisse les années suivantes : 3,98 millions en 1938, 2,48 millions en 1939 et 419939 en 1940 (ROGNANT, Loïc, Types de régions touristiques en Italie. Essai de macrogéographie, thèse de doctorat d'État en géographique, Université de Nice, 1981, p. 314).

${ }^{7}$ BOSWORTH, Richard J., "The Touring Club Italiano and the Nationalization of the Italian Bourgeoisie », in European History Quartely, 3/1997, pp. 373-375 ; PIVATO, Stefano, Il Touring Club Italiano, Bologna, Il Mulino, 2006.

${ }^{8}$ GARZARELLI, Benedetta, «Fascismo e propaganda all'estero : le origini della direzione generale per la propaganda (1933-1934)», in Studi storici, 1/2002, pp. 477-520.

${ }_{9}^{9}$ PALOSCIA, Franco, Storia del turismo nell'economia italiana, cit. ; TIZZONI, Elisa, « Les politiques touristiques $\mathrm{du}$ fascisme et les relations internationales de l'Italie, entre diplomatie publique et création d'une marque destination-Italie ", in Cahiers de la Méditerranée, 88, 2/2014, pp. 85-98; ID., "Politica e turismo in epoca fascista tra centro e periferia », in Ricerche di storia politica, 2/2017, pp. 147-168.
} 


\section{Adapter les équipements touristiques aux ambitions fascistes et au bien- être des touristes}

Dans le but de pérenniser les flux touristiques en Italie et de diffuser une image favorable du régime auprès des étrangers, les dirigeants fascistes prennent conscience dès les années vingt de la nécessité d'adapter les équipements de la péninsule aux exigences des touristes.

C'est la légère baisse du nombre de visiteurs étrangers à la fin de la décennie, en comparaison avec le Royaume-Uni, la France et l'Allemagne, qui motive la rédaction d'un premier rapport de cinq pages, en juillet 1929. Son auteur, le sénateur Romeo Gallenga, sans donner de chiffres précis $^{10}$, estime qu'une nouvelle politique est nécessaire pour y remédier dans le but de prouver au monde le bon fonctionnement de l'industrie italienne, de ses services publics et l'adhésion de la population au fascisme ${ }^{11}$. Il déplore particulièrement que les étrangers des autres continents qui viennent en Europe, principalement les Américains, préfèrent se rendre à Londres, Paris, ou Berlin, plutôt qu'en Italie. Les explications qu'il avance sont diverses mais la cause principale reste selon lui les « campagnes calomnieuses des fuorusciti, des maçons, des socialistes et des juifs». Au-delà de l'accusation des ennemis affichés du régime et de l'affirmation d'un antisémitisme notoire, Gallenga donne quelques pistes pour accroître les flux de touristes. La situation des hôtels par exemple, qui selon lui sont trop chers pour les voyageurs et dont les gérants refusent de baisser les prix à cause de taxes jugées trop lourdes, doit encourager le gouvernement à modifier la fiscalité et à changer le système des surtaxes et des pourcentages qui s'ajoutent au prix et que les visiteurs ne comprennent pas. Il assure aussi que le manque de distractions est un regret souvent émis et qu'il est nécessaire d'y remédier par une publicité accrue dans la presse étrangère pour présenter les spectacles, les divertissements ou les sports qu'il est possible de pratiquer en Italie.

Ce premier rapport, peu approfondi, est suivi un an plus tard d'un second plus prolixe. Composé de dix-huit pages de texte et de quarante-quatre pages de statistiques, il est rédigé en décembre 1930 par Fulvio Suvich, ancien sous-secrétaire d'État aux Finances de novembre 1926 à juillet 1928 et président de l'ENIT depuis un $a^{12}$. Lui aussi directement adressé à Mussolini, il est qualifié par son auteur de « strictement confidentiel » parce qu'il traite des efforts à accomplir en

\footnotetext{
${ }^{10}$ En 1926 et en 1927, 1,2 million de touristes étrangers visitent l'Italie, dont une majorité d'Allemands (226 343 en 1926 et 273565 en 1927), d'Américains (197 783 en 1926 et 225585 en 1927) et d'Anglais (183 342 en 1926 et 171562 en 1927) : ENIT, Statistica del movimento turistico in Italia. 1926-1927, Roma, Castaldi, 1929, pp. 68, 70. En 1928, une baisse est observée par les autorités italiennes sur l'ensemble du mouvement des voyageurs (245 801 Allemands, 205711 Américains et 155510 Anglais) : ENIT, Statistica del Movimento turistico in Italia. 1928, Roma, Castaldi, 1930, pp. 30-31.

${ }^{11}$ Rapport du 10 juillet 1929 à la présidence du Conseil : Archivio Centrale dello Stato (ACS), Presidenza del Consiglio dei Ministri (PCM), 1928-1930, busta 1187, fasc. 3/2-9, protocollo 7990.

${ }^{12}$ Rapport de décembre 1930 au chef du gouvernement, ibidem.
} 
matière de tourisme. Il est dans ce sens très éclairant quant aux orientations concrètes du régime. Le lancement de la «bataille du tourisme » quelques mois plus tôt ${ }^{13}$, dont le terme même rappelle celui de la «bataille du blé ", évoque l'importance que revêt le tourisme pour l'État, notamment avec l'internationalisation de la crise économique. Les premières pages présentent les sources de financement de l'ENIT, ses orientations futures et l'organisation de la corporation du tourisme. Sont ensuite exposés les problèmes soulevés par les infrastructures touristiques, puis les propositions pour améliorer le sort des voyageurs en privilégiant les équipements et les prix, la qualité des routes et du transport ferroviaire, l'harmonisation des règlements aux frontières et le développement du tourisme dans les provinces ayant subi la guerre, ainsi qu'en Libye ${ }^{14}$.

Les établissements de tourisme qui servent à loger les étrangers de passage font l'objet des premières remarques. Suvich estime notamment que le confort et les prix sont à améliorer, particulièrement pour les bourses moyennes ${ }^{15}$, et que trop de régions demeurent mal équipées. Les territoires qui présentent selon lui l'offre la plus variée sont les régions montagneuses, notamment le Haut-Adige et le Val d'Aoste, prouvant l'intérêt des touristes pour l'essor des sports d'hiver, mais aussi l'importance des flux en provenance des pays limitrophes, l'Allemagne, l'Autriche, la Suisse ou la France. Concernant le tourisme balnéaire, l'inquiétude vient d'abord de la Riviera italienne, moins bien dotée que sa voisine française, et qui pâtit par conséquent de ses sous-équipements. Il est vrai que le développement extraordinaire de la Côte d'Azur depuis la fin du XIX ${ }^{e}$ siècle a attiré les touristes du monde entier, notamment les plus fortunés ${ }^{16}$. Les hôtels sont confortables et les palaces célèbres, même si la crise de 1929 a provoqué une plus grande rareté de la clientèle internationale et certaines fermetures ${ }^{17}$. L'ouverture progressive des établissements l'été puis la création des congés payés en 1936 ont néanmoins permis de faire face aux difficultés en aggravant la concurrence avec les installations italiennes ${ }^{18}$. Globalement, les autorités fascistes ont bien conscience du retard de l'Italie dans le domaine de l'hôtellerie, que les chambres sont souvent chères par rapport aux prestations et que l'image du régime qui en résulte est regrettable. Le 6 avril 1930, le secrétaire général de l'ENIT, Angelo Mariotti, avait déjà proposé lors d'une conférence de refonder le système hôtelier pour accroître la clientèle, persuadé de la nécessité de l'adapter aux attentes des voyageurs ${ }^{19}$. Ces inquiétudes sont d'autant plus légitimes que dans les guides touristiques éditées chez Hachette par le Touring Club italien, à l'instar du

\footnotetext{
${ }^{13}$ GARINI, G., « La battaglia del turismo », in Ospitalità italiana, 8, 1929, pp. 77-78.

${ }^{14}$ Ce dernier point ne sera pas étudié ici.

${ }^{15}$ Un décret le 21 février 1932 instaurera la publication obligatoire des prix dans les hôtels qui seront désormais fixés sur la base d'un rapport annuel remis à l'ENIT.

${ }^{16}$ BOYER, Marc, L'invention de la Côte d'Azur. L'hiver dans le Midi, La Tour d'Aigue, Éditions de l'Aube, 2002.

${ }^{17}$ Trois siècles de tourisme dans les Alpes-Maritimes, catalogue de l'Exposition organisée par le Conseil général aux archives départementales, Milano, Silvana Editoriale, 2013, pp. 50-59.

${ }^{18}$ Ibidem, pp. 104-118.

${ }^{19}$ MARIOTTI, Angelo, L'importanza economica del turismo, conferenza detta al R. Istituto Superiore di Scienze Economiche e Commerciali a Firenze il 6 aprile 1930, Firenze, AESTI, 1931.
} 
Guide bleu bien diffusé en France, si les remarques sur les hôtels sont globalement bienveillantes, elles sont parfois accompagnées d'observations plus négatives que quelques avis sur la qualité de la nourriture cherchent à nuancer. Dans l'édition de 1932, qui reprend mot à mot les précédentes, il est énoncé que « dans les grandes villes touristiques, l'hôtellerie est excellente, avec électricité, bains, eau courante chaude et froide, chauffage... Dans les villes plus petites, les hôtels sont généralement moins satisfaisants et le degré de confort, que l'on peut s'attendre à y trouver, varie beaucoup entre un établissement et un autre. Toutefois, la cuisine y est souvent excellente, quoique sans prétention, et les vins, du cru local, sont généralement exquis $"^{20}$. Le guide allemand Baedeker donne pour sa part des renseignements sur les prix des hôtels sans faire preuve de critique excessive sur l'état des établissements, préférant s'attarder davantage sur la nourriture : « à l'hôtel, on refusera les chambres au nord, sans soleil, et on s'assurera que les couvertures de lit sont suffisamment chaudes. Pour la nourriture et la boisson, il faudra être prudent; on s'abîmerait aisément l'estomac si on le chargeait de mets inaccoutumés tels que macaroni à l'italienne, fromages, fruit du Midi, etc. Pour le poisson, on imitera les Italiens qui refusent celui qui n'est pas frais; il faudra s'abstenir à Naples et à Venise de manger des huîtres, celles-ci pouvant occasionner la fièvre typhoïde $»^{21}$.

Les impressions des voyageurs sur le sujet varient beaucoup en fonction de leurs moyens financiers et de la région qu'ils visitent. Dans les années vingt, elles sont plutôt négatives. Le pèlerin André Mabille de Poncheville décrit près de Viterbe une chambre excessivement sale qui l'a empêché de fermer l'œil22. De même, à Catanzaro en Calabre, Hélène Tuzet qui conduit pour la Fondation Rockefeller une enquête sur les écoles de l'Italie méridionale, regrette être descendue dans un hôtel «indiciblement malpropre »: «Je n'osais toucher aux serviettes de toilette, qui avaient déjà servi Dieu sait à quels voyageurs, où l'on me regardait comme un animal sauvage et où l'on s'obstinait à ne me parler que par des gestes " ${ }^{23}$. Au début des années trente, de l'avis du journaliste Émile Schreiber, après un long séjour en URSS, ou de sa consœur Paule Herfort, toute acquise au régime fasciste, les hôtels italiens sont de très bonne qualitée ${ }^{24}$. Cependant, les critiques acerbes existent aussi. En 1934, le touriste Eugène Grangié écrit à propos de l'une de ses chambres: «La porte ferme mal, comme il arrive partout en Italie. Le mobilier est ancien et défraîchi, les murs tâchés, le tapis élimé, la toilette et ses accessoires quelconques » ${ }^{25}$. À Turin en

\footnotetext{
${ }^{20}$ Les Guides bleus. L'Italie en un volume, Paris, Hachette, 1932, p. XIX.

${ }^{21}$ BAEDEKER, Karl, L'Italie des Alpes à Naples, Leipzig-Paris, Agence générale de librairie et de publications, 1926, p. XII. Les éditions suivantes font globalement les mêmes commentaires.

${ }^{22}$ MABILLE DE PONCHEVILLE, André, Le Chemin de Rome. Feuilles de route, Paris, Bloud et Gay, 1926, p. 188.

${ }^{23}$ TUZET, Hélène, Calabre et Sicile : une enquête en 1928, présentation de Leonardo Sciascia, Moncalieri-Genève, Slatkine-CIRVI, 1985, p. 81.

${ }^{24}$ SCHREIBER, Émile, Rome après Moscou, Paris, Plon, 1932, p. 5 ; HERFORT, Paule, Chez les Romains fascistes, préface d'Henry Bérenger, Paris, Éditions de la Revue mondiale, 1934, p. 211.

${ }^{25}$ GRANGIÉ, Eugène, Deux semaines en Italie (printemps 1934), Cahors, Coueslant, 1934, p. 113.
} 
1938, le reporter Émile Condroyer explique qu'il loge dans un hôtel aux murs lézardés, tandis que les torchons et les draps qui pendent aux balcons, ainsi que les gargouillements d'évier, lui font dire que «la vieille Italie» existe encore ${ }^{26}$. À lire ces reproches, on mesure les difficultés du régime fasciste à imposer un confort minimum. Concernant la gastronomie, là encore les contradictions sont de mise. L'écrivain Jean-Louis Vaudoyer considère dans les années vingt que le royaume de Victor-Emmanuel est le pays où l'on mange le mieux après la France ${ }^{27}$, alors qu'Eugène Grangié, dix ans plus tard, assure avoir été déçu par la nourriture italienne. Il se plaint dans son récit de voyage de consommer toujours les mêmes aliments, pâtes et rôti de veau, épinards en branches et pommes de terre, fromage et orange, même s'il trouve les vins excellents $^{28}$. Le touriste Pierre Clerget regrette aussi une nourriture peu variée ${ }^{29}$. Au contraire, le journaliste Maurice Ferrus qui traverse plusieurs régions d'Italie en 1938 dit apprécier l'alimentation, en particulier la viande rôtie, alors qu'il avait entendu qu'on ne mangeait que des spaghetti et du bœuf bouilli partout dans le royaume ${ }^{30}$.

Le deuxième axe de réflexion du rapport Suvich concerne les établissements de divertissement que l'auteur juge trop méconnus par les touristes, ces derniers se plaignant trop souvent de voyager dans un pays ennuyeux, ce que le rapport Gallenga mettait du reste déjà en évidence. Suvich considère ces accusations infondées en raison des nombreuses installations sportives, des théâtres ou des boîtes de nuit qui existent dans les principaux lieux touristiques. Pourtant, les remarques des voyageurs sont à ce sujet sans équivoque. Après la publication du rapport, tous continuent à dépeindre un pays où les cafés sont vides le soir et les distractions rares ${ }^{31}$. Simone de Beauvoir et Jean-Paul Sartre, visitant Rome au cours de l'été 1933, sont particulièrement déçus par une ville qui, la nuit tombée, devient déserte, laissant place aux seuls miliciens qui patrouillent pour exiger des touristes qu'ils aillent se coucher ${ }^{32}$. Pour remédier à ces critiques, Suvich souhaite que les pouvoirs publics se concentrent sur le développement des casinos. Néanmoins, le régime qui a condamné les jeux de hasard les a supprimés, à l'instar de celui de Venise, si bien que seul celui de San Remo demeure important pour contrebalancer l'influence de Monte-Carlo et de la Riviera française, sans toutefois y parvenir. Dans ce domaine, l'État italien ne parvient pas à dépasser ses contradictions.

\footnotetext{
${ }^{26}$ CONDROYER, Émile, « Promenades italiennes. De l'élégance, encore de l'élégance », in Le Journal, 3 octobre 1938 , p. 3.

${ }^{27}$ VAUDOYER, Jean-Louis, Les délices de l'Italie, Paris, Plon, 1924, p. 181.

${ }^{28}$ GRANGIÉ, Eugène, Deux semaines en Italie (printemps 1934), cit., pp. 111-112.

${ }^{29}$ CLERGET, Pierre, Sur les routes de Savoie et d'Italie, Lyon, Imprimerie du Salut Public, 1932, p. 16.

${ }^{30}$ FERRUS, Maurice, Impressions d'Italie, Bordeaux, Imprimerie Pechade, [s.d.], p. 18.

${ }^{31}$ RECOULY, Raymond, L'Italie fasciste, Grenoble, Arthaud, 1934, p. 37 ; FLAMENT, Albert, « Tableaux de Rome ", in Revue de Paris, 15 novembre 1935, p. 457 ; SCHNEIDER, Édouard, Dans Rome vivante, Paris, Plon, 1936, p. 230.

${ }^{32}$ DE BEAUVOIR, Simone, La Force de l'âge, Paris, Gallimard, 1960, p. 161.
} 
Les infrastructures de transport sont une autre importante préoccupation pour Suvich. Essentielles pour l'acheminement et le déplacement des touristes, leur amélioration doit permettre de diffuser une image renouvelée de la péninsule, dans un pays où pendant des décennies les voyageurs se sont plaints des retards de train ou de leur inconfort ${ }^{33}$. Les chemins de fer sont donc une priorité du régime et Suvich juge obligatoire l'électrification des lignes, l'embellissement des gares, l'amélioration de leurs services (latrines, buffets, porteurs) et précise qu' «il serait opportun - et c'est une petite chose mais qui donne l'impression de la bonne éducation de tout un peuple - que les toilettes soient munies comme à l'étranger de tout le nécessaire ». L'électrification des lignes est en réalité un effort constant depuis l'arrivée de Mussolini au pouvoir (5 218 kilomètres réalisés en 1940, contre 780 en 1923) ${ }^{34}$. Les temps de parcours n'ont cessé également de se réduire grâce à la rapidité croissante des trains et à l'amélioration du réseau. Si en 1926 il fallait onze heures pour faire Milan-Rome et 36 heures pour aller de Milan en Sicile ${ }^{35}$, en 1938, il ne faut plus que huit heures trente pour le premier trajet et 22 heures pour le second ${ }^{36}$. Concernant la ponctualité des trains, Suvich n'en parle pas. En lisant les récits des voyageurs, le changement semble pourtant radical à tous, faisant même admettre aux plus antifascistes que dans ce domaine, l'autoritarisme semble bénéfique ${ }^{37}$. Ce silence laisse penser que ce problème ancien, pour Suvich, est considéré comme définitivement réglé. Celui-ci salue également les efforts du régime concernant l'aménagement des routes, devenu nécessaire en raison de l'essor du trafic automobile. Pour poursuivre la modernisation, il ambitionne de renforcer le partenariat entre l'ENIT et l'Azienda Autonoma della Strada, créée en 1928, et estime nécessaire d'augmenter les commodités le long des principales voies, à l'instar des cabines téléphoniques, des postes de secours ou des indications de garages et d'hôtels. En outre, immédiatement après le rapport, le régime continue à macadamiser de nombreux tronçons du réseau routier, prouvant qu'il poursuit la politique entamée depuis plusieurs années ${ }^{38}$. Si les guides touristiques saluent les efforts accomplis, comme le Baedeker qui regrette malgré tout que les routes demeurent poussiéreuses et boueuses après la plui $e^{39}$, les voyageurs, globalement, ne tarissent pas non plus d'éloges. Émile Schreiber en 1932 révèle «le prodigieux effort réalisé

\footnotetext{
${ }^{33}$ MILZA, Pierre, Français et Italiens à la fin du XIX siècle, t. I, Rome, École Française de Rome, 1981, p. 320.

${ }^{34}$ MAGGI, Stefano, Storia dei trasporti in Italia, Bologna, Il Mulino, 2005, p. 52.

${ }^{35}$ Les Guides bleus. Premier volume des Alpes à Rome, Paris, Hachette, 1926, p. LII.

${ }^{36}$ ROGNANT, Loïc, Types de régions touristiques en Italie, cit., p. 309.

${ }^{37}$ NAUDEAU, Ludovic, L'Italie fasciste ou l'autre danger, Paris, Flammarion, 1927, p. 17 ; BÉRAUD, Henri, Ce que j'ai vu à Rome, Paris, Éditions de France, 1929, pp. 156-157 ; BORDEAUX, Henry, La Claire Italie, Paris, Plon, 1929, p. 7 ; BERG DE BREDA, Souvenirs de Rome et d'Italie, Compiègne, Progrès de l'Oise, 1927, p. 103 ; BANCAL, Léon, L'Italie et nous, Marseille, Imprimerie du Petit Marseillais, 1932, p. 55 ; LUCAIN, Marcel, Les Romains d'aujourd'hui, Paris, Tallandier, 1933, p. 170.

${ }^{38}$ MATHIEU, Georges, Contribution à l'étude économique de l'Italie en 1932, Alger, Imprimerie Baconnier Frères, 1933, p. 37.

${ }^{39}$ BAEDEKER, Karl, Italie centrale et Rome, Leipzig-Paris, Agence générale de librairie et de publications, 1929, p. 15.
} 
depuis dix ans dans ce pays dont la médiocrité des voies de communication semblait un mal irrémédiable $»^{40}$. Tout au long des années trente, des remarques similaires abondent dans les comptes rendus de séjours ${ }^{41}$.

La dernière grande ambition du régime évoquée par Suvich est le désir de valoriser les visites des champs de bataille de la Grande Guerre. Il affirme que présenter la ligne de front telle qu'elle était pendant le conflit aurait un grand intérêt patriotique, éducatif et touristique, sans oublier les retombées économiques qui faciliteraient les reconstructions des provinces ayant souffert des combats. En 1930 pourtant, il regrette qu'aucune organisation ne propose ce type de visite en pointant du doigt le retard de l'Italie dans ce domaine, notamment par comparaison avec la France $^{42}$. Suvich propose dans un premier temps de recenser les zones qui peuvent être conservées et remises en état, avant de stabiliser les itinéraires pour rejoindre les zones de front, d'améliorer les moyens de transports pour s'y rendre, de préparer des guides en différentes langues avec des historiques, de créer des panneaux explicatifs sur place et de développer une propagande touristique sur le sujet, à l'étranger comme en Italie, notamment dans les localités à proximité des anciens lieux d'affrontements, à l'instar de Venise. Sa préférence, pour commencer, va à la mise en valeur du territoire de Gorizia qui offre selon lui l'avantage d'avoir à proximité le cimetière de Redipuglia. Un aménagement permettra effectivement, en septembre 1938, d'en faire le plus grand cimetière militaire du monde, avec les restes de 100000 soldats morts pendant la guerre, et d'y accueillir de nombreux visiteurs. Cependant, durant les années qui suivent le rapport, en dépit des souhaits de Suvich, les brochures touristiques italiennes qui sont diffusées à l'étranger demeurent peu prolixes concernant les visites des champs de bataille et pour les voyageurs, hormis les militaires ou les anciens combattants, la zone de front n'apparaît jamais comme un but de séjour.

Ainsi, le régime fasciste qui a encadré toutes les activités touristiques à l'aube des années trente a aussi parallèlement cherché à adapter son offre à ses ambitions et aux commentaires de ses visiteurs. Il a également dû se préoccuper des problématiques liées au passage de la frontière et à la surveillance, afin de concilier dictature totalitaire et flux touristiques.

\footnotetext{
${ }^{40}$ SCHREIBER, Émile, Rome après Moscou, cit., p. 14.

${ }^{41}$ GEORGES-MICHEL, Michel, Italia...Italia, Paris, Baudinière, 1934, p. 240 ; GRANGIÉ, Eugène, Deux semaines en Italie (printemps 1934), cit., p. 41 ; BAC, Ferdinand, Promenades dans l'Italie nouvelle. Rome, Compiègne, Hachette, 1933, p. 145 ; CLERGET, Pierre, Sur les routes de Savoie et d'Italie, cit., pp. 6, 35.

${ }^{42}$ Sur le tourisme de guerre, voir l'ouvrage pionnier de LLOYD, David Wharton, Battlefield tourism. Pilgrimage and the commemoration of the Great War in Britain, Australia and Canada, 1919-1939, Oxford, Berg, 1998. Pour le cas français, voir les contributions de BRANDT, Susanne, «Le voyage au champ de bataille », in Vingtième siècle. Revue d'histoire, 41, 1/1994, pp. 18-22, WINTER, Caroline, « Tourism, social memory and the Great War », in Annals of Tourism Research, 36, 4/2009, pp. 607-626 ; HERTZOG, Anne, « Tourisme de mémoire et imaginaire touristique des champs de bataille », in Via. Tourism Review, 1, 2012, URL: < http://journals.openedition.org/viatourism/1276 > [consulté le 9 novembre 2018].
} 


\section{Simplifier le passage de la frontière et amoindrir la surveillance}

Les touristes qui voyagent en Italie sont dès le passage de la frontière confrontés aux contraintes de la dictature. Elles se traduisent principalement par des contrôles excessifs des passeports ou des bagages et une surveillance omniprésente. Dès lors, le régime, pour pérenniser son attractivité sans abandonner la lutte antifasciste et la construction totalitaire, doit concilier ces contraintes au bon accueil des hôtes étrangers.

Conscient de l'image néfaste que les contrôles abusifs aux frontières peuvent donner aux étrangers, Romeo Gallenga, dès juillet 1929 dans son rapport à la présidence du Conseil, émet le souhait qu'ils soient moins rigoureux et que les déclarations d'état-civil à remplir dans les hôtels soient moins contraignantes ${ }^{43}$. En décembre 1930, Fulvio Suvich exprime les mêmes ambitions en proposant des solutions concrètes ${ }^{44}$. Elles sont le fruit d'une lettre, qualifiée de "confidentielle ", qu'il a envoyée le 25 septembre à Arturo Bocchini ${ }^{45}$, chef de la police du régime, et qui est jointe à son rapport. Les contacts pris entre les organes touristiques et les services de police lui ont semblé essentiels afin, qu'ensemble, ils collaborent pour éviter les critiques des touristes qui dégradent l'image du régime ou qui pourraient les faire fuir. Dans cette lettre, Suvich insiste d'abord sur le manque de clarté des panneaux installés dans les localités proches de la frontière et servant à avertir le public que les agents de surveillance ont ordre de tirer sur quiconque tenterait de la passer illégalement. Il serait selon lui plus judicieux de placer sur les pancartes les signalisations exactes des passages ouverts, avec l'indication des documents nécessaires pour se rendre en Italie, tout en avertissant qu'en cas de traversée par les versants non autorisés, le touriste peut faire l'objet de graves sanctions. Suvich soulève ensuite la question de la lenteur des opérations de contrôle aux frontières qui créent, assure-t-il, trop de protestations. Il refuse d'incriminer le manque d'effectifs, attestant que les gardes frontières des pays voisins ne sont pas plus nombreux. En revanche, il dénonce clairement un manque de rationalisation des tâches et le problème des délais occasionnés par les recherches de personnes suspectes pour le régime, sur les listes spéciales, donnant l'impression au touriste d'être soumis à une surveillance insupportable. En outre, à peine la frontière franchie, d'autres contrôles de papiers d'identité ou de bagages peuvent avoir lieu par la milice, les services des douanes ou les carabiniers, alors qu'ils semblent inutiles et qu'ils sont source d'exaspération pour celui qui les subit. Suvich dénonce aussi le comportement des agents de surveillance qui manquent trop souvent d'amabilité, affirmant en

\footnotetext{
${ }^{43}$ Rapport du 10 juillet 1929 à la présidence du Conseil, op. cit. L'omission de remplir une déclaration de séjour, obligatoire pour tous les voyageurs qui arrivent en Italie, peut conduire à des sanctions ou à des arrestations.

${ }^{44}$ Rapport de décembre 1930 au chef du gouvernement, op. cit.

${ }^{45}$ CARAFOLI, Domizia, PADIGLIONE, Gustavo, Il Viceduce. Storia di Arturo Bocchini capo della polizia fascista, Milano, Rusconi, 1987.
} 
avoir été informé à plusieurs reprises au col du Brenner ou à Vintimille : «c'est un inconvénient qui devrait être éliminé avec une certaine facilité parce que la gentillesse ne coûte rien et fait une meilleure impression, spécialement dans un État fort comme l'est généralement considérée l'Italie ». Toutes ces observations lui semblent si fondamentales qu'elles sont directement reprises dans le rapport qu'il envoie à Mussolini en décembre. Il y rappelle la situation des voyageurs qui perdent beaucoup trop de temps lors des contrôles, soit à se faire comprendre, soit à cause des formalités trop lourdes auxquelles ils doivent se soumettre. Donner l'impression à chaque touriste, quand il entre dans la péninsule, "qu'il est une espèce de délinquant », lui semble inadmissible, persuadé que les contrôles devraient être effectués par «des fonctionnaires intelligents et de bon sens ", pour les réduire au minimum. Comme le passage de la frontière est le premier contact d'un voyageur avec le régime fasciste, sa première impression sur l'Italie «nouvelle », il doit être facilité parce qu'il est déterminant pour la suite du séjour. Le but, selon Suvich, est d'éviter la diffusion à l'étranger de propos indésirables sur un régime qui imposerait persécutions, injustices et surveillance abusive dès l'entrée sur son territoire. Il estime donc indispensable de mieux coordonner les services et d'harmoniser les sévères dispositions douanières avec les intérêts touristiques en exigeant des douaniers, comme des carabiniers, qu'ils se montrent plus courtois avec les touristes et qu'ils connaissent quelques mots des langues les plus courantes. Il émet également le désir que la tenue des agents soit plus soignée car si les carabiniers sont, dit-il, irréprochables, ce n'est pas le cas de tous les miliciens. En outre, la proposition de l'ENIT de donner des cours spéciaux au personnel affecté dans les zones frontalières, pour lui enseigner comment se vêtir ou comment s'adresser aux touristes, est à ses yeux une excellente mesure qui doit être généralisée. Reste enfin une dernière question qui est abordée, celle de l'interdiction des appareils photographiques près des frontières, à l'origine de nombreux incidents. Le sujet est particulièrement délicat parce que beaucoup de voyageurs ne connaissent pas cette législation, si bien que la confiscation systématique semble à Suvich inadaptée. Les vérifications, pense-t-il, « doivent être fait[e]s avec la juste mesure et avec bon sens, quand il s'agit manifestement de touristes qui ne sont pas mal intentionnés ».

Toutes ces considérations sont largement confirmées par les témoignages des voyageurs, à commencer par le problème du passage de la frontière dans les zones montagneuses, là où elle est mal délimitée. Les dossiers de touristes arrêtés après l'avoir malencontreusement franchie sont si nombreux dans les archives diplomatiques qu'ils confirment largement les propos de Suvich. En mai 1924 par exemple, deux promeneurs en randonnée au-dessus de Menton, dont l'un d'eux possédait un appareil photographique, sont arrêtés au Mont Grammont par des douaniers italiens et conduits à la caserne de Vintimille pour y être interrogés ${ }^{46}$. Le sommet, qui offre à près de 1400

\footnotetext{
${ }^{46}$ Note non signée du 20 mai 1924 écrite de Menton et adressée au ministère des Affaires étrangères :
} 
mètres d'altitude une vue imprenable sur la zone frontière, est particulièrement stratégique et les autorités italiennes préfèrent vérifier les intentions des deux Français, après leur avoir confisqué leur pellicule. Le Quai d'Orsay s'émeut de cette sévère surveillance exercée à la frontière italienne où les soupçons d'espionnage pour raisons politiques, économiques ou militaires sont de plus en plus courants ${ }^{47}$. Le 29 juin 1927, c'est un certain Louis Cochard qui est arrêté dans un refuge du versant italien du Mont Viso, alors qu'il attend un guide transalpin, sous prétexte que son passeport est périmé depuis le 3 juin, qu'il n'a pas de visa italien, qu'il possède un couteau d'une dimension interdite en Italie, une carte d'état-major française, une carte routière Michelin de la région et qu'il est capitaine de réserve dans l'aviation ${ }^{48}$. Les conditions de son arrestation et de sa détention sont particulièrement violentes. Les carabiniers se montrent brutaux à son égard. Ils l'enferment à la prison de Saluces en lui donnant pendant une semaine la ration attribuée aux détenus de droit commun ${ }^{49}$. Le consul de France à Turin, informé tardivement de l'événement, alors que l'article 90 de la loi de Sécurité publique, approuvée par le décret royal du 8 novembre 1899, demande aux préfectures du royaume de prévenir immédiatement les consulats intéressés

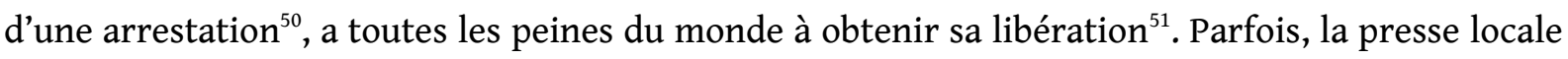
a connaissance de certaines mésaventures. L'histoire du docteur Reignaud et de l'employé des Chemins de fer Joly, dans les Alpes-Maritimes, que diffuse Le Petit niçois à la fin du mois d'août 1927, est particulièrement illustrative. Partis en randonnée au cours de l'été, ils se sont égarés à travers les éboulis près du fort de l'Authion, non loin du col de Tende, et se sont retrouvés par mégarde en territoire italien lorsque ont surgi des carabiniers qui ont examiné leurs passeports, les ont conduits à un poste frontière qui se situait à deux heures de marche, où des fascistes les ont fouillés, avant de se voir imposés une nouvelle marche vers le poste de Saint-Dalmas ${ }^{52}$. Ce type d'incident, qui ne pouvait qu'alimenter critiques et calomnies sur l'Italie à l'étranger, continue tout au long des années trente malgré les avertissements et les propositions de Suvich. Ils sont même parfois dramatiques. En juillet 1938, deux touristes qui se promènent dans la région d'Abriès, dans les Hautes-Alpes, franchissent la frontière au cours d'une excursion et cinq ou six

Archives du ministère des Affaires étrangères (AMAE), Correspondance politique et commerciale (19141940), Z-Europe, Italie (1918-1929), carton 219.

${ }^{47}$ Ibidem.

${ }^{48}$ Aide-mémoire du 29 juillet 1927 de l'ambassade de France à Rome pour le ministère royal des Affaires étrangères : Archivio storico del ministero degli Affari esteri (ASMAE), Affari Politici, Francia (1919-1930), busta 1108, fasc. 4054 .

${ }^{49}$ Ibidem.

${ }^{50}$ Circulaire de mai 1927 du ministère de l'Intérieur envoyée à tous les préfets du royaume : ACS, ministero dell'Interno, direzione generale della Pubblica sicurezza, affari generali e riservati, massime (1880-1954), busta 27 , fasc. 9 .

${ }^{51}$ Lettre du 2 août 1927 du chargé d'affaires à l'ambassade de France à Rome au ministre des Affaires étrangères: AMAE, Correspondance politique et commerciale (1914-1940), Z-Europe, Italie (1918-1929), carton 219.

${ }^{52}$ Le Petit niçois, 31 août 1927, pp. 1-2. 
mètres plus loin essuient des coups de feu des carabiniers, avant de se replier et d'être poursuivis à l'intérieur du territoire français, où les tirs continuent et finissent par blesser l'un d'entre eux ${ }^{53}$.

Les touristes qui franchissent la frontière par les grandes routes ou les voies ferrées confirment également les propos de Suvich. Avant son rapport et celui du sénateur Gallenga, les contrôles successifs, la rigueur des agents et la lenteur des procédures sont récurrents. Lors de l'Année Sainte 1925, où les pèlerins sont particulièrement nombreux à se rendre en Italie, les plaintes concernant les contrôles des billets de train, l'inspection des bagages et les attentes interminables sont permanentes ${ }^{54}$. Ces contrôles très stricts se renforcent même par la suite à cause de la tentative d'attentat contre Mussolini de l'anarchiste Gino Lucetti, le 11 septembre $1926^{55}$. Un an plus tard, le commissaire spécial de la ville de Menton fait en effet part au ministère de l'Intérieur de l'autorité avec laquelle le nouvel inspecteur de la sûreté publique, qui vient d'être nommé à Vintimille, a exigé de ses fonctionnaires la plus grande fermeté ${ }^{56}$. Dans toute l'Italie, les douaniers font désormais preuve d'un tel zèle que les voyageurs manquent parfois leur train à cause de procédures infinies imposées par le régime ${ }^{57}$. Si l'on ajoute à ces difficultés les confiscations régulières d'appareils photographiques, que les touristes considèrent aléatoires, on mesure mieux les préoccupations de Suvich. Suite à son rapport, une plus grande collaboration entre le Commissariat pour le tourisme et la direction générale de la Pubblica sicurezza est décidée, permettant dès la fin de l'année 1931 l'amélioration des services de contrôle à l'entrée du royaume $^{58}$. Elle semble porter ses fruits car durant les années suivantes, les voyageurs témoignent de réels changements. Tous soulignent à plusieurs reprises la courtoisie des douaniers et des contrôles sans complication majeure $\mathrm{e}^{59}$. Le 20 mars 1934, Eugène Grangié qui passe la frontière à

\footnotetext{
${ }^{53}$ Télégramme du 5 juillet 1938 du ministère des Affaires étrangères au chargé d'affaires à l'ambassade de France à Rome : AMAE, Correspondance politique et commerciale (1914-1940), Z-Europe, Italie (1918-1929), carton 393.

${ }^{54}$ BAZOT, Georges, Mon voyage d'Italie (avril-mai 1925), Avignon, Aubanel Frères, 1925, p. 1 ; GUÉRY, Charles, En auto-car par les Alpes, la Toscane, l'Ombrie, Rome, les Salines, la Riviera, etc. Du 7 au 27 septembre 1925, Évreux, Imprimerie de l'Eure, 1926, p. 11; WEITER, Pierre, Le pèlerinage lorrain à Rome en septembre 1925, Metz, Imprimerie lorraine, 1926, p. 21.

${ }^{55}$ MASSARD, Émile, "L'état des esprits en Italie. "C'est la faute à la France" ", in Le Matin, 17 septembre 1926, p. 1.

${ }^{56}$ Lettre du 13 septembre 1927 du directeur de la sûreté générale du ministère de l'Intérieur au ministre des Affaires étrangères, qui transmet un rapport du 6 août 1927 du commissaire spécial de la ville de Menton : AMAE, op. cit., carton 219.

${ }^{57}$ Cette aventure est vécue en septembre 1927 par un groupe de pèlerins qui débarque à Livourne et qui ne peut profiter du premier train à cause des nombreuses formalités douanières : DONCCEUR, Paul, Troisième carnet de route. Roumieux. Pèlerins d'Assise et de Rome, Paris, Imprimerie-édition de l'Art catholique, 1927, pp. 19-20.

${ }^{58}$ Note du 16 décembre 1931 du Commissariat pour le tourisme au chef du gouvernement : ACS, PCM, 19311933, busta 1504, fasc. 3/2-1, protocollo 3804 .

${ }^{59}$ SCHREIBER, Émile, Rome après Moscou, cit., pp. 2-3 ; DEFRANCE, J., Souvenirs d'un voyage en Italie, imprimé par Robert Ferric à Bolbec, 1933, p. 6 ; Souvenir du premier pèlerinage d'Arpajon à Rome. 26 février-9 mars 1934, Belley, Chaduc, 1934, p. 2; LUYS, Georges, Croisière dans l'archipel toscan à bord du "Caducée", Orléans, Imprimerie du Loiret, 1935, p. 7.
} 
Menton écrit : « La consigne est de sourire au touriste qui rarement passe de la contrebande et qui toujours apporte du bel argent neuf, bon à recevoir $»^{60}$. Si quelques voyageurs regrettent toujours les «lenteurs habituelles» et d'«inévitables complications pour le visa des passeports et les perquisitions de la douane $»^{61}$, ainsi que le spectacle de douaniers qui présentent «un air terriblement sévère" dans la zone frontière ${ }^{62}$, ils ne sont plus majoritaires. Cependant, la dégradation des relations franco-italiennes à partir de 1936 conduit à nouveau à des remarques déplaisantes ${ }^{63}$.

Parallèlement aux contraintes inhérentes au passage de la frontière, la surveillance est aussi régulièrement dénoncée par des touristes qui ont le sentiment d'être épiés en permanence ${ }^{64}$. Le contrôle très régulier de leurs billets de train est en particulier un moyen pour le régime de suivre précisément l'évolution de leurs itinéraires et de s'assurer de leur identité ${ }^{65}$. Cependant, toutes les mesures prises se révèlent toujours désagréables et les plaintes adressées à l'ambassade de France pour les dénoncer sont nombreuses ${ }^{66}$. Si les autorités italiennes assurent qu'elles sont toujours discrètes, elles regrettent parfois des comportements abusifs. C'est le cas en décembre 1931 lorsque le Commissariat pour le tourisme avoue que deux jeunes mariés en voyage de noces, de nationalité française, fortement irrités par les « continuels et agaçants contrôles » qui leur ont été imposés dans presque toutes les villes dans lesquelles ils ont fait halte, ont finalement décidé d'écourter leur séjour et de rentrer en France ${ }^{67}$. Il résulte de ces attitudes une image dégradée du régime. Le journaliste Pierre Scize l'illustre de manière très ironique en avril 1936. Il écrit que l'Italie de Mussolini est toujours le paradis des touristes si l'on excepte « la surveillance policière à peine déguisée, l'écoute au téléphone, l'espionnage des garçons d'hôtel, portiers, guides, chauffeurs $»^{68}$. Contrairement à ces propos, avant comme après le rapport Suvich, des voyageurs

\footnotetext{
${ }^{60}$ GRANGIÉ, Eugène, Deux semaines en Italie (printemps 1934), cit., p. 11.

${ }^{61}$ PIÉTU, A., Un pèlerinage à Rome, Châteauroux, Imprimerie centrale, 1934, p. 14.

${ }^{62}$ FOBIS, Henri-Jean, Mon premier voyage à Rome, Niort, L'Action Intellectuelle, 1936, p. 29.

${ }^{63}$ MARTIN DU GARD, Roger, Journal II 1919-1936, Paris, Gallimard, 1993, p. 1210 ; PÉCOUL, Georges, Deux promenades en Corse. Une semaine en Italie, Paris, Imprimerie Tancrède, 1938, p. 217.

${ }^{64}$ BERG DE BREDA, Souvenirs de Rome et d'Italie, cit., p. 103 ; LAURENT, Fernand, " Impressions d'Italie. Après l'attentat contre M. Mussolini ", in Le Petit Parisien, 20 septembre 1926, p. 1 ; MARTIN, Georges, "L'Italie après cinq ans de fascisme. Au pays du ciel bleu et des chemises noires. Le consul et ses licteurs ", in Le Petit Journal, 15 octobre 1927, p. 2 ; DORSAY, «Un français moyen dans l'Italie de 1936 », in Je suis partout, 12 septembre 1936, p. 1 .

${ }^{65}$ Sur le régime policier, voir : FRANZINELLI, Mimmo, I tentacoli dell'Ovra. Agenti, collaboratori e vittime della polizia politica fascista, Torino, Bollati Boringhieri, 1999.

${ }^{66}$ En septembre 1926 par exemple, Germaine Marguerite Coster, professeur de dessin de la ville de Paris, signale au consul de France à Cagliari « les mesures de surveillance excessive dont elle a été l'objet en Sardaigne [...] de la part des policiers et des carabiniers » : lettre du 2 septembre 1926 du consul de France à Cagliari à l'ambassadeur à Rome, Centre des archives diplomatiques de Nantes, archives de l'ambassade de France à Rome-Quirinal, carton 198.

${ }^{67}$ Note du 16 décembre 1931 du Commissariat pour le tourisme pour le chef du gouvernement: ASMAE, op. cit., busta 1104, fasc. 4003.

${ }^{68}$ SCIZE, Pierre, « L'Italie ? J'en viens », in Voilà, 25 avril 1936, p. 6.
} 
attestent que la surveillance, sans la nier, n'est jamais oppressante et qu'elle demeure discrète ${ }^{69}$. Roger Martin du Gard, qui séjourne à Florence en février 1937, admet même jouir en Italie d'une totale liberté. Il se justifie: "Aucune de mes lettres n'a été ouverte. J’ai reçu des journaux de gauche librement. La discipline n'a aucun caractère de brimade. Les agents qui l'appliquent le font intelligemment; et la plupart des gens préfèrent au désordre cette réglementation acceptée, expliquée, nullement tyrannique $»^{70}$.

En somme, si le régime fasciste a multiplié les mesures pour éviter aux touristes les contraintes de la dictature, à lire leurs remarques, bien des limites sont perceptibles, en dépit des efforts accomplis. Pour le régime, imposer une image avantageuse demeure une priorité et cela commence par la qualité de l'accueil dans ses bureaux touristiques à l'étranger.

\section{Offrir une image irréprochable du régime fasciste dans ses bureaux} touristiques à l'étranger

Pour le gouvernement fasciste, l'image du redressement de l'Italie doit passer par l'exemplarité de ses bureaux touristiques à l'étranger, dont la visite est le premier contact d'un voyageur avec la péninsule. Or, au début des années trente, des dysfonctionnements majeurs dans celui de la CIT à Paris, ouvert depuis 1927, inquiètent au plus au point les autorités italiennes.

L'affaire commence au début de l'été 1933 lorsque deux rapports anonymes sont directement envoyés à Mussolini pour l'alerter sur la situation. Le premier, daté du 24 juin, insiste sur les plaintes des touristes français concernant le fonctionnement du bureau parisien ${ }^{71}$. Si ce dernier vient d'intégrer de nouveaux locaux en raison de l'inconfort, du désordre et de la mauvaise organisation des services dans les anciens espaces, les défaillances semblent demeurer, à commencer par la systématique alternance des employés : «Il ne se passe pas une semaine, il ne se passe pas un mois, sans qu'au comptoir ne se présentent des visages nouveaux $»^{72}$. Le rapport dénonce également l'irrégularité du fonctionnement du service d'informations, avec des horaires

\footnotetext{
${ }^{69}$ NAUDEAU, Ludovic, L'Italie fasciste ou l'autre danger, cit., p. 19 ; HELSEY, Édouard, « Une enquête du Journal. L'Italie a l'homme qu'elle voulait ", in Le Journal, 4 décembre 1926, p. 1 ; LESOURD, Paul, Impressions romaines, Paris, Spes, 1929, pp. 14-15; AUBERT, Henry, Indiscrétions et curiosités sur l'Italie, Paris, E. Messein, 1931, p. 10 ; CHRISTOPHE, Pierre, Florete flores. En souvenir du pèlerinage de l'A.C.J.F. à Rome, Épinal, Imprimerie Coopérative, 1934, p. 37 ; SOUVENANCE, Jean, Retour d'Italie fasciste. Impressions et réflexions, Herblay, L'Idée Libre, 1938, p. 14 ; FERRUS, Maurice, Impressions d'Italie, cit., pp. 9-10.

${ }^{70}$ Lettre du 7 février 1937 à Jean Blanzat : MARTIN DU GARD, Roger, Correspondance générale, t. VII, 1937-1939, Paris, Gallimard, 1992, pp. 27-28.

${ }^{71}$ Copie du rapport parvenu au chef du gouvernement et envoyé le 4 septembre 1933 par le Commissariat pour le tourisme au ministre des Communications : ACS, PCM, 1931-1933, busta 1504, fasc. 3/2-1, protocollo 11356.

${ }^{72}$ Une mention dans la marge du rapport indique que cette phrase a été soulignée par Mussolini dans le document original.
} 
inexacts et des indications erronées qui entraînent bon nombre de réclamations de la part du public. La qualité de l'accueil pose aussi problème : «L'indiscipline de la part du personnel est un autre motif d'observations et de commentaires peu élogieux» pour des agents qui sont jugés discourtois, peu serviables, aux connaissances linguistiques insuffisantes et dont le faible salaire, selon l'auteur, pourrait expliquer le manque de motivation. Un exemple concret est développé pour illustrer le propos. Il est notifié qu'un jour, un sénateur français s'est rendu au bureau de la CIT pour retirer un billet pour une parente et qu'il s'est plaint du manque de sollicitude de la part des employés, davantage occupés à plaisanter qu'à assurer leur service. En outre, les discussions politiques de certains agents au comptoir, qui se vantent de ne pas appartenir aux organisations fascistes, sont dénoncées. Une femme qui était venue demander des informations sur la Mostra della rivoluzione fascista ${ }^{73}$ s'était par exemple aussitôt vue répondre qu'il y avait des choses plus intéressantes à voir à Rome. Toutes ces critiques, bien qu'anonymes, ne peuvent qu'irriter les autorités fascistes, d'autant qu'un second rapport, lui aussi non signé et sans date, probablement envoyé à Mussolini à la même période que le premier, évoque plus précisément les reproches des touristes français enregistrés auprès du bureau parisien de la $\mathrm{CIT}^{74}$. Il incrimine particulièrement les erreurs d'informations reçues par les employés, notamment concernant les réductions des billets de train, qui ont pu faire renoncer des voyageurs prêts à partir.

Ces diverses dénonciations sont prises très au sérieux par le régime italien qui décide de commanditer une enquête en septembre 1933, afin d'en connaître le bien-fondé. Une commission voit le jour, composée d'Amedeo Fani, ancien sous-secrétaire d'État aux Affaires étrangères, nommé par le secrétaire du Parti fasciste, de Giovanni Talvacchia, désigné par le sous-secrétaire d'État à la présidence du Conseil, et de Luigi Fiori, conseiller d'administration des Chemins de fer de l'État, nommé par le ministère des Communications. Elle rend son rapport le 29 septembre à Galeazzo Ciano, responsable du bureau de presse de Mussolini, qui le fait parvenir au Duce le 2 octobre $^{75}$. Fulvio Suvich a d'abord été contacté en sa qualité de Commissaire général pour le tourisme et comme sous-secrétaire d'État aux Affaires étrangères, afin de savoir s'il avait eu connaissance d'autres écrits de ce genre. Il a répondu par la négative et a déclaré qu'il ne fallait pas donner trop d'importance à ces deux rapports anonymes. À Paris, toutes les personnes compétentes susceptibles de fournir des informations ont été interrogées. Le chargé d'affaires auprès de l'ambassade d'Italie a certifié n'avoir jamais reçu de réclamations concernant le bureau de la CIT, aussi bien de la part d'Italiens que de Français. Le conseiller commercial de la même

\footnotetext{
${ }^{73}$ Il s'agit de l'Exposition de la Révolution fasciste, inaugurée le 28 octobre 1932 pour les dix ans du régime et qui a accueilli près de quatre millions de visiteurs étrangers: GENTILE, Emilio, La religion fasciste. La sacralisation de la politique dans l'Italie fasciste, Paris, Perrin, 2002, p. 225.

${ }^{74}$ Copie du rapport envoyé par le télégramme sans date du sous-secrétaire d'État aux Affaires étrangères au Commissariat pour le tourisme : ACS, PCM, 1931-1933, busta 1504, fasc. 3/2-1, protocollo 11356.

${ }^{75}$ Il fait dix pages et comprend quatre annexes : ibidem.
} 
ambassade a fait des observations similaires. Il a ajouté que les employés envoyés récemment à Paris, pour répondre aux modifications récentes du bureau, n'ont sans doute pas encore acquis toute la pratique nécessaire pour ce travail et qu'il se peut qu'il y ait eu des erreurs. Le consul général Camerani a aussi exclu avoir reçu une quelconque plainte. Il a néanmoins confirmé que les employés étaient généralement très jeunes et peu payés. Le secrétaire administratif du Fascio a affirmé que ses relations avec le personnel, dont les membres sont presque tous inscrits dans l'organisation, étaient bonnes. Plusieurs autres témoins ont également été auditionnés, comme le président de la Chambre de commerce italienne de Paris, et toutes ont attesté ne jamais avoir reçu de reproches à propos des bureaux de la CIT. La commission d'enquête a surtout eu un long entretien avec Ilario Zamuner, le directeur du bureau parisien. Celui-ci a pris connaissance des accusations et s'est rapidement montré surpris, soutenant avoir reçu de plusieurs personnes des éloges sur sa gestion. Tous ces témoignages ont pu rassurer les membres de la commission, lors de leur retour à Rome. Leur rapport conclut du reste que le bureau de la CIT à Paris fonctionne convenablement. Il confirme les propos de la journaliste Paule Herfort, certes très favorable au régime, qui écrit à la même période : « Le tourisme est la grande préoccupation italienne. Il suffit de regarder le luxe et la merveilleuse organisation des bureaux touristiques de la péninsule, à Paris, pour se convaincre de l'effort fait dans ce sens $»^{76}$.

Cette affaire révèle l'intérêt spécifique que le gouvernement fasciste accorde au bon fonctionnement de ses agences touristiques à l'étranger. Elles sont une vitrine de l'Italie, si bien que leur personnel doit être irréprochable. La mise en place d'une commission d'enquête, suite à deux témoignages anonymes, le prouve. Plus généralement, l'ensemble des rapports étudiés établit que le tourisme a bien été une priorité pour le régime, même si conjuguer bien-être des touristes et contraintes de la dictature n'a pas toujours été chose aisée, comme les remarques des voyageurs l'illustrent. Pour le gouvernement fasciste, la politique touristique a été en permanence un véritable défi afin de surmonter deux objectifs en apparence contradictoires : pérenniser les flux de visiteurs en donnant l'image la plus favorable possible du régime sans renoncer à la construction totalitaire qui s'accélère dans les années trente. Si jusqu'en 1939 l'Italie demeure une destination touristique importante, favorisant la propagande mussolinienne et servant son économie, les critiques récurrentes des voyageurs à propos des contraintes de la dictature, même de la part des plus bienveillants, montre l'impossibilité de concilier une image positive du fascisme avec les ambitions touristiques affichées dès les années vingt.

\footnotetext{
${ }^{76}$ HERFORT, Paule, Chez les Romains fascistes, cit., p. 211.
} 


\section{L'AUTEUR}

Christophe POUPAULT est professeur d'histoire en classes préparatoires aux grandes écoles et à Sciences po Aix. Parmi ses dernières publications : À l'ombre des faisceaux. Les voyages français dans l'Italie des chemises noires, Rome, Collection de l'École française de Rome, 2014 et en codirection, Voyager dans les États autoritaires et totalitaires de l'Europe de l'entre-deuxguerres: confrontations aux régimes, perceptions des idéologies et comparaisons, Chambéry, Éditions de l'Université Savoie Mont Blanc, 2017.

URL: < http://www.studistorici.com/progett/autori/\#Poupault > 